\title{
Helicity and the SOLIS Vector-Spectromagnetograph
}

\author{
Christoph U. Keller, John W. Harvey, Carl J. Henney \\ National Solar Observatory, 950 N. Cherry Ave., Tucson, AZ 85719, \\ USA
}

Harrison P. Jones

NASA/Goddard Space Flight Center, 950 N. Cherry Ave., Tucson, AZ 85719 , USA

\begin{abstract}
The SOLIS Vector-Spectromagnetograph (VSM) is a compact, highly efficient vector-polarimeter that measures the magnetic field vector over the full solar disk within 12 minutes. Helicity-related quantities will be derived from the observed vector field.
\end{abstract}

\section{Overview}

SOLIS (Synoptic Optical Long-term Investigations of the Sun) is a suite of three innovative instruments that greatly improve ground-based synoptic solar observations. The VSM is a $50-\mathrm{cm}$ effective aperture telescope with a tip-tilt secondary mirror and filled with helium to reduce internal seeing. The spectrograph has a Littrow design that is insensitive to temperature changes. The entrance slit is scanned in declination to provide 2048 by 2048 pixel scans of the full solar disk at three wavelengths. The polarization modulation is performed by ferroelectric liquid crystal retarders. Two 1024 by 256 CMOS-Hybrid cameras acquire 92 frames per second, which are then analyzed to obtain all Stokes parameters. The VSM is capable of recording vector magnetograms in two FeI lines around $630.2 \mathrm{~nm}$, deep longitudinal magnetograms in the same lines, longitudinal magnetograms in CaII $854.2 \mathrm{~nm}$, and intensity in HeI 1083.0 $\mathrm{nm}$. Regular full-disk scans will be recorded within 12 minutes, while active regions can be scanned in as little as 30 seconds. The Stokes spectra are reduced to physical quantities including field strength, azimuth, inclination, and filling factor with a Milne-Eddington inversion code. The VSM will be the prime instrument for synoptic studies of helicity in the solar photosphere. In addition, the SOLIS Full-Disk Patrol (FDP) will provide the velocity vector to measure the flux of magnetic helicity through the photosphere. More information on SOLIS is available at solis.nso.edu.

\section{Acknowledgments}

The National Solar Observatory is operated by AURA Inc. under cooperative agreement with the National Science Foundation. 